\title{
Digestibilité des protéines et disponibilité des acides aminés de quelques matières premières chez le bar (Dicentrarchus labrax)
}

\author{
P. SPYRIDAKIS, J. GABAUDAN, R. METAILLER, J. GUILLAUME
}

IFREMER Centre de Brest, B.P. 7029263 Plouzané, France.

Summary. Protein digestibility and amino acid availability of some feed ingredients for European sea bass (Dicentrarchus labrax).

Protein digestibility of soyabean meal, casein-gelatin mixture and fish hydrolysate was tested in the European sea bass, using increasing substitution levels in a reference diet. The protein digestibility of the latter composed of Norwegian fish meal had already been established (91\%).

An accurate estimation of the apparent digestibility coefficient was made by linear regression, for the casein-gelatin mixture and soyabean meal, but not for fish hydrolysate (non significant correlation, $\mathrm{p}>0.05$ ).

Protein digestibility estimated by extrapolation varied with the substitution level. The estimations obtained with the highest level used for each ingredient were close to those obtained by linear regression. However they exhibited a high variability for soyabean meal and, to a lesser extent, for fish hydrolysate.

The availability of individual amino acids was also investigated in each protein source. For some amino acids it was different from that of total nitrogen, suggesting that the availability of essential amino acids should be used for accurate feed formulation.

Metabolic fecal nitrogen and amino acids were determined using a protein-free diet, in order to estimate true availabilities. These coefficients were not significantly different from apparent availability coefficients.

\section{Introduction.}

La digestibilité protéique de diverses matières premières n'a fait l'objet que de rares études chez le bar (Alliot, 1982 ; Alliot et al., 1976, 1978). Des données supplémentaires sont nécessaires pour la détermination des besoins en protéines et en acides aminés et pour la formulation d'aliments plus performants. La mesure de la digestibilité protéique d'un régime dont les protéines proviennent d'une source autre que la farine de poisson s'avère difficile pour des raisons d'appétence ou même de formulation quand les matières premières contiennent peu de protéines. La digestibilité des différentes sources est donc estimée par substitution partielle, généralement de l'ordre de $30 \%$ (Cho e/ al., 1982, 1985), dans un 
régime de référence de digestibilité connue, et extrapolation à $100 \%$ à partir des valeurs obtenues pour le mélange et le régime de référence. Cette méthode suppose que la digestibilité varie linéairement en fonction du taux d'incorporation et que la variabilité des mesures soit faible.

Nombre de matières premières, susceptibles d'être utilisées en alimentation des poissons, sont déficientes en certains acides aminés. II est indispensable de connaître la disponibilité de ces derniers, afin de pouvoir couvrir les besoins du poisson en acides aminés essentiels lors de la formulation des aliments.

La présente étude a été menée afin de vérifier chez le bar la validité du calcul de digestibilité de trois sources protéiques par extrapolation avec un seul ou plusieurs taux de substitution et d'établir la disponibilité des acides aminés pour ces matières premières.

\section{Matériel et méthodes.}

Des juvéniles de bar d'un poids moyen de $50 \mathrm{~g}$, élevés au laboratoire, ont été acclimatés à $18^{\circ} \mathrm{C}$ dans des bacs cylindroconiques de 40 I (20 poissons par bac) pendant trois semaines avant le début de l'expérience.

La composition des régimes utilisés est donnée dans le tableau 1. Le régime de référence est à base de farine de poisson norvégienne dont la digestibilité apparente, établie à plusieurs reprises, est de $91 \%$. La digestibilité protéique d'un tourteau de soja, d'un mélange caséine-gélatine et d'un hydrolysat de poisson a été testée par substitution à taux croissants dans le régime de référence. Toutefois, la composition de ce dernier a été légèrement modifiée afin de garder les mêmes rapports entre nutriments pour l'ensemble des régimes. Tous les aliments contiennent approximativement $45 \%$ de protéines, $12 \%$ de lipides et $15 \%$ de glucides. Les aliments ont été distribués sous forme de granulés humides (environ $30 \%$ d'eau) de $3 \mathrm{~mm}$ de diamètre.

Un régime sans protéines (remplacement de la farine de poisson par un mélange cellulose-amidon dans un rapport de 60/40) a été utilisé pour quantifier l'excrétion de l'azote endogène et calculer la digestibilité réelle. Ce dernier régime a été administré par gavage à la suite d'un refus systématique d'ingestion volontaire par les poissons. La composition en acides aminés des régimes au taux d'incorporation le plus élevé, pour une source donnée, figure dans le tableau 2.

Les fèces ont été recueillies par filtration en continu de l'eau (Choubert et al., 1982) et selon la procédure décrite par Spyridakis et al. (1988).

L'azote des aliments et des fèces a été dosé en duplicat par la méthode de Kjeldahl et l'oxyde de chrome en triplicat par la méthode de Bolin et al. (1952), sur des échantillons lyophilisés.

Le dosage des acides aminés a été effectué, après hydrolyse acide $(\mathrm{HCl}, 6 \mathrm{~N}$, $4 \mathrm{~h}, 155^{\circ} \mathrm{C}$ ), par chromatographie liquide (analyseur Chromakon 400) selon la méthode de Moore et al. (1958). Des dosages d'acides aminés de l'albumine de bœuf ont montré un taux de récupération élevé (de l'ordre de $95 \%$ ) sauf pour la méthionine et la cystine. Ces derniers, ainsi que le tryptophane, complètement détruit, ne sont pas présentés dans cette étude. Les coefficients d'utilisation 
TABLEAU 1

Composition des régimes expérimentaux.

\begin{tabular}{|c|c|c|c|c|}
\hline \multirow{2}{*}{ Ingrédients } & \multicolumn{4}{|c|}{ Régimes $\left({ }^{1}\right)$} \\
\hline & $\mathrm{FP}$ & CG & TS & HP \\
\hline $\begin{array}{l}\text { Farine de poisson }\left({ }^{2}\right) \\
\text { (Prot. } 70 \% \text {, Lip. } 10 \% \text { ) }\end{array}$ & 64,3 & $51,4-12,9$ & $51,4-25,7$ & $51,4-25,7$ \\
\hline Caséine (Prot. $92 \%$ ) & - & $7,4-29,7$ & - & - \\
\hline Gélatine (Prot. $90 \%$ ) & - & $2,4-9,6$ & - & - \\
\hline $\begin{array}{l}\text { Tourteau de soja } \\
\text { (Prot. } 47 \% \text {, Lip. } 1 \% \text { ) }\end{array}$ & - & - & $19,1-57,4$ & - \\
\hline $\begin{array}{l}\text { Hydrolysat de poisson }\left({ }^{3}\right) \\
\text { (Prot. } 85 \% \text {, Lip. } 5 \% \text { ) }\end{array}$ & - & - & - & $10,6-31,8$ \\
\hline Huile de foie de morue & 4,6 & $5,9-9,7$ & $5,7-7,9$ & $5,3-6,8$ \\
\hline Amidon prégélatinisé & 15,0 & 15,0 & $9,3-0 \quad(*)$ & 15,0 \\
\hline Cellulose & 8,65 & $10,45-15,65$ & $7,05-1,55$ & $10,25-13,25$ \\
\hline Alginate & 4,0 & 4,0 & 4,0 & 4,0 \\
\hline Mélange vitaminique $\left({ }^{4}\right)$ & 1,0 & 1,0 & 1,0 & 1,0 \\
\hline Vitamine $\mathrm{C}$ & 0,15 & 0,15 & 0,15 & 0,15 \\
\hline Chlorure de choline & 0,3 & 0,3 & 0,3 & 0,3 \\
\hline Lécithine de soja & 1,0 & 1,0 & 1,0 & 1,0 \\
\hline $\mathrm{Cr}_{2} \mathrm{O}_{3}$ & 1,0 & 1,0 & 1,0 & 1,0 \\
\hline Protéines $(N \times 6,25)$ & 45,2 & $45,1-46,6$ & $45,4-46,1$ & $44,8-45,6$ \\
\hline Lipides & 12,2 & $12,3-12,6$ & $11,8-12,6$ & $12,6-13,2$ \\
\hline
\end{tabular}

( $\left.{ }^{1}\right)$ Substitution, à taux croissants, des protéines du régime de référence à base de farine de poisson norvégienne (FP) par: Mélange de caséine-gélatine (CG) (20 à $80 \%$ ); Tourteau de soja (TS) $(20$ à $60 \%)$; Hydrolysat de poisson (HP) $(20$ à $60 \%)$.

$\left({ }^{2}\right)$ Norseamink. ND
$\left({ }^{3}\right)$ CPSP 90 Anorel. ${ }^{\text {ND }}$
(4) Par kg de mélange :

( $\left.{ }^{4}\right)$ Par $\mathrm{kg}$ de mélange: Vit. A acétate, 1000000 UI ; Vit. $D_{3}, 100000$ UI; Vit. E acétate, $4000 \mathrm{mg}$; Vit. $K_{3}, 100 \mathrm{mg}$; Vit. B1, $1000 \mathrm{mg}$; Vit. B2, $2500 \mathrm{mg}$; D Pantothenate de calcium, $5000 \mathrm{mg}$; Vit. B6, $1000 \mathrm{mg}$; Vit. B12, $6 \mathrm{mg}$; Vit. PP, $10000 \mathrm{mg}$; Acide folique, $500 \mathrm{mg}$; Biotine, $100 \mathrm{mg}$; Méso-inositol, $100000 \mathrm{mg}$. Support fin de protéine de soja traitée.

(*) Apport de glucides par le tourteau de soja, estimé à $30 \%$.

digestive apparente (CUDA) et réelle (CUDR) (Kim, 1974) ont été calculés selon les formules:

$$
\begin{aligned}
& \text { CUDA }=100-100 \times(\mathrm{CA} / \mathrm{NA} \times \mathrm{Nf} / \mathrm{Cf}) \\
& \text { CUDR }=100 \times \frac{\mathrm{NA} / \mathrm{CA}-\left(\mathrm{Nf} / \mathrm{Cf}-\mathrm{N}^{\prime} \mathrm{f} / \mathrm{C}^{\prime} \mathrm{f}+\mathrm{N}^{\prime} \mathrm{a} / \mathrm{C}^{\prime} \mathrm{a}\right)}{\mathrm{NA} / \mathrm{CA}}
\end{aligned}
$$

$\mathrm{CA}: \% \mathrm{Cr}_{2} \mathrm{O}_{3}$ dans l'aliment; $\mathrm{NA}$ : \% nutriment dans l'aliment ; $\mathrm{Cf}: \% \mathrm{Cr}_{2} \mathrm{O}_{3}$ dans les fèces; $\mathrm{Nf}$ : \% nutriment dans les fèces; $\mathrm{C}^{\prime} \mathrm{a}: \% \mathrm{Cr}_{2} \mathrm{O}_{3}$ dans l'aliment protéiprive ; $\mathrm{N}^{\prime} \mathrm{a}$ : \% nutriment dans l'aliment protéiprive ; $\mathrm{C}^{\prime} \mathrm{f}: \% \mathrm{Cr}_{2} \mathrm{O}_{3}$ dans les fèces provenant du régime protéiprive ; $N^{\prime} f$ : \% nutriment dans les fèces provenant du régime protéiprive. 
Composition en acides aminés des régimes utilisés (pour les taux d'incorporation les plus élevées par source)

\begin{tabular}{ccccc}
\hline & \multicolumn{5}{c}{ Régimes } \\
\cline { 2 - 5 } $\begin{array}{c}\text { Acides aminés } \\
\text { (\% m.s. })\end{array}$ & $\begin{array}{c}\text { FP } \\
\text { N }\end{array}$ & CG & TS & HP \\
ASP & $400 \%$ & $60 \%$ & $60 \%$ \\
THR & 2,52 & 4,10 & 5,44 & 4,66 \\
SER & 1,66 & 1,80 & 2,15 & 1,94 \\
GLU & 6,38 & 1,86 & 2,24 & 1,69 \\
PRO & 1,90 & 7,39 & 7,00 & 5,73 \\
GLY & 2,67 & 4,35 & 1,89 & 2,13 \\
ALA & 2,98 & 3,01 & 2,18 & 3,20 \\
VAL & 2,41 & 2,23 & 2,30 & 2,85 \\
ILE & 2,33 & 2,41 & 2,16 & 2,04 \\
LEU & 3,62 & 2,16 & 2,17 & 2,06 \\
TYR & 1,87 & 3,34 & 3,39 & 3,02 \\
PHE & 2,10 & 1,76 & 1,57 & 1,37 \\
HIS & 1,38 & 1,90 & 2,10 & 1,81 \\
LYS & 3,52 & 1,23 & 1,20 & 1,01 \\
ARG & 2,92 & 3,20 & 3,01 & 3,19 \\
& 2,58 & 2,97 & 2,64 \\
\hline
\end{tabular}

Les intervalles de confiance (IC) de la digestibilité protéique des matières premières pour chaque taux de substitution ont été calculés selon la formule suivante dérivée de celle de Yoshida (1972) pour le calcul de l'erreur standard:

$$
\text { IC matière première }=\sigma_{r} \times \sqrt{\frac{1+(1-\alpha)^{2}}{\alpha}} \times t_{0,5}
$$

$\alpha(0$ à 1$)$ : taux d'incorporation; $\sigma_{\mathrm{r}}$ : écart-type de la mesure sur le régime.

\section{Résultats.}

La digestibilité protéique apparente des différents régimes, en fonction de la source et du taux d'incorporation, est représentée sur la figure 1. Elle augmente avec le niveau de substitution pour le mélange caséine-gélatine tandis qu'elle diminue pour le tourteau de soja. La digestibilité des régimes contenant de I'hydrolysat de poisson est voisine de celle du régime de référence. II est à noter que l'erreur standard de la mesure des coefficients de digestibilité tend à augmenter avec le taux d'incorporation pour le tourteau de soja et dans une moindre mesure pour l'hydrolysat de poisson. Les CUDA des trois sources testées, calculés par extrapolation, soit à partir d'un seul taux de substitution, soit en utilisant l'ensemble des taux par source, sont donnés dans le tableau 3. La régression entre le CUDA et le taux d'incorporation est linéaire pour le mélange caséine-gélatine $(r=0,97)$ et pour le tourteau de soja $(r=0,94)$. Elle est apparemment curvilinéaire pour l'hydrolysat de poisson, l'équation quadratique obtenue étant la suivante: $Y=91,60+0,78 x-0,001 x^{2}$. Toutefois, le coefficient de corrélation n'est significatif $(p>0,05)$ ni avec l'ajustement linéaire $(r=-0,25)$ ni avec l'ajustement curvilinéaire $(R=-0,24)$. 


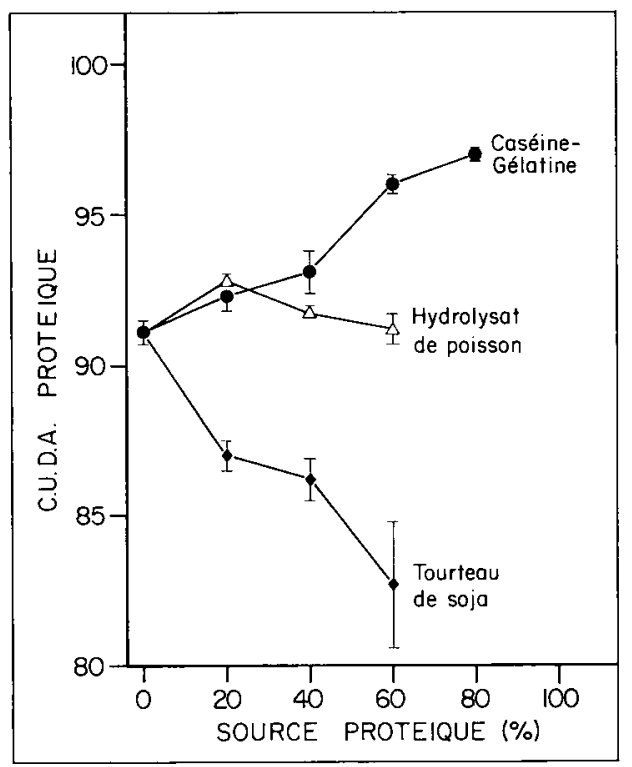

FIG. 1. - Coefficient d'utilisation digestive apparente protéique des régimes testés en fonction du taux de substitution par source.

\section{TABLEAU 3}

Coefficient d'utilisation digestive apparente des protéines (moyenne sur quatre déterminations) et intervalle de confiance des estimations.

\begin{tabular}{|c|c|c|c|c|c|c|}
\hline \multirow{2}{*}{$\begin{array}{l}\text { Mode de calcul } \\
\text { Taux } \\
\text { de substitution (\%) }\end{array}$} & \multicolumn{4}{|c|}{ Extrapolation ( $\left.{ }^{1}\right)$} & \multirow[t]{3}{*}{$\begin{array}{l}\text { Régression } \\
\text { linéaire }\end{array}$} & \multirow[b]{3}{*}{$r$} \\
\hline & 20 & 40 & 60 & 80 & & \\
\hline Source & & & & & & \\
\hline Casé & $1 \pm 12,0$ & $96,1 \pm 10,3$ & $99,3 \pm 12,0$ & $98,5 \pm 1,7$ & $98,6 \pm 2,2$ & $+0,97$ \\
\hline Tourteau de soja & $70,6 \pm 12,9$ & $78,9 \pm 11,2$ & $77,1 \pm 24,9$ & 二 & $77,7 \pm 4,5$ & $-0,94$ \\
\hline Hydrolysat de poisson & $99,6 \pm 5,2$ & $92,6 \pm 1,7$ & $91,3 \pm 6,9$ & - & $91,4 \pm 2,4$ & $-0,25$ \\
\hline
\end{tabular}

( ${ }^{1}$ ) Intervalle de confiance calculé à l'aide de la formule citée en Matériel et Méthodes.

La disponibilité apparente et réelle des acides aminés et la digestibilité de l'azote total du régime de référence et des trois sources testées, ainsi que la composition des fèces en acides aminés après ingestion du régime protéiprive, sont données dans le tableau 4. Le CUDA de l'azote total ainsi que celui de la somme des acides aminés essentiels ou totaux n'est pas différent du CUDR correspondant. 
TABLEAU 4

Coefficients d'utilisation digestive apparente et réelle des acides aminés et de l'azote total du régime de référence et des trois sources testées (moyenne sur quatre déterminations) et composition en acides aminés fécaux d'origine endogène.

\begin{tabular}{|c|c|c|c|c|c|c|c|c|c|}
\hline \multirow{2}{*}{$\begin{array}{l}\text { Acides } \\
\text { aminés }\end{array}$} & \multicolumn{2}{|c|}{ FP } & \multicolumn{2}{|c|}{ CG } & \multicolumn{2}{|c|}{ TS } & \multicolumn{2}{|c|}{ HP } & \multirow{2}{*}{$\begin{array}{c}\text { AAFE } \\
\text { (mg/100 g } \\
\text { aliment) }\end{array}$} \\
\hline & CUDA & CUDR & CUDA & CUDR & CUDA & CUDR & CUDA & CUDR & \\
\hline ASP & 82,2 & 81,8 & 99,6 & 99,1 & 81,5 & 81,0 & 93,9 & 93,3 & 78 \\
\hline THR & 88,3 & 89,7 & 98,7 & 100,5 & 74,3 & 76,5 & 94,0 & 95,9 & 63 \\
\hline SER & 86,9 & 87,4 & 99,4 & 99,8 & 75,9 & 76,4 & 94,2 & 94,9 & 35 \\
\hline GLU & 90,2 & 89,5 & 99,5 & 98,6 & 81,7 & 80,8 & 95,0 & 93,8 & 109 \\
\hline PRO & 90,3 & 90,4 & 99,9 & 99,9 & 75,8 & 76,1 & 95,0 & 95,1 & 57 \\
\hline GLY & 87,6 & 88,2 & 99,1 & 99,7 & 74,4 & 75,4 & 95,4 & 95,9 & 66 \\
\hline ALA & 88,8 & 88,7 & 98,7 & 98,6 & 73,3 & 73,4 & 95,3 & 95,2 & 48 \\
\hline VAL & 86,9 & 86,5 & 99,4 & 99,1 & 73,7 & 73,3 & 89,7 & 89,3 & 32 \\
\hline ILE & 88,0 & 87,5 & 97,4 & 96,8 & 68,8 & 68,2 & 90,3 & 89,5 & 37 \\
\hline LEU & 89,4 & 88,9 & 99,2 & 98,5 & 71,1 & 70,2 & 91,4 & 90,6 & 63 \\
\hline TYR & 93,5 & 93,1 & 99,6 & 99,2 & 73,8 & 72,8 & 90.7 & 89,9 & 16 \\
\hline PHE & 89,0 & 88,0 & 98,8 & 97,5 & 66,5 & 65,2 & 89,3 & 87,7 & 32 \\
\hline LYS & 93,0 & 92,2 & 100,0 & 99,0 & 87.3 & 86,2 & 94,0 & 92,9 & 14 \\
\hline HIS & 92,6 & 90,9 & 99,2 & 97,2 & 80.3 & 77,4 & 86,3 & 82,7 & traces \\
\hline ARG & 92,3 & 91,7 & 99,8 & 99,2 & 82,1 & 81,4 & 95,5 & 94,7 & 35 \\
\hline AAE & 89,9 & 89,2 & 99,2 & 98,2 & 74,2 & 73,2 & 92,2 & 91,0 & \\
\hline AAT & 89,0 & 88,6 & 99,4 & 99,0 & 77,2 & 76,8 & 93,3 & 92,6 & \\
\hline $\begin{array}{l}\text { Azote } \\
\text { total }\end{array}$ & 91,1 & 91,2 & 98,5 & 98,6 & 77.1 & 77,2 & 91,3 & 91,4 & \\
\hline
\end{tabular}

AAE : Acides aminés essentiels; AAT: Acides aminés totaux; AAFE : Acides aminés fécaux d'origine endogène.

\section{Discussion.}

Les résultats de la présente étude montrent que l'estimation de la digestibilité théorique, calculée par extrapolation à partir de chaque taux de substitution, varie en fonction de ce dernier. La variabilité des mesures diminue avec l'augmentation du taux d'incorporation dans le cas du mélange caséine-gélatine, ce qui s'explique aisément d'après la formule de Yoshida (1972) pour les cas où l'écart-type du CUDA des régimes est constant. Ce n'est pas le cas pour le tourteau de soja, ni pour l'hydrolysat de poisson ; pour ces matières premières, l'écart-type du CUDA des régimes est plus grand pour les quantités élevées de ces ingrédients. Dans ces cas l'augmentation de précision que l'on pouvait espérer est vraisemblablement annulée par un phénomène d'ordre biologique, la moins bonne « tolérance " de la matière première quand elle est incorporée à forte dose.

Le calcul du CUDA par régression sur l'ensemble des données fournit une bonne estimation pour deux sources de protéines, le mélange caséine-gélatine et le tourteau de soja, les régressions étant linéaires et les coefficients de corrélation élevés. On peut noter que l'estimation du CUDA par régression diffère très peu de celle obtenue par extrapolation à partir du taux le plus élevé.

Dans le cas de l'hydrolysat de poisson, l'absence de corrélation significative entre les CUDA des protéines du régime et le taux d'incorporation de l'ingrédient rend difficile la discussion de la variation observée. 
Ces résultats appellent des commentaires sur plusieurs points : quand la digestibilité protéique varie linéairement en fonction du taux d'incorporation de l'ingrédient à tester, et que l'écart-type de la mesure reste constant, on obtient une bonne estimation, soit avec la méthode de régression linéaire, soit avec une extrapolation à un seul taux, pourvu que celui-ci soit élevé ; on peut noter que, avec nos techniques, le taux de $30 \%$ couramment employé ne permet qu'une faible précision. Quand l'écart-type des mesures s'accroît avec le niveau d'incorporation, les conclusions sont moins évidentes : l'estimation par extrapolation à partir du taux de $60 \%$ paraît proche de celle obtenue par régression; toutefois l'erreur standard est relativement élevée, ce qui prouve que l'estimation n'est pas très fiable.

Indépendamment de la méthode de calcul, les CUDA obtenus sont supérieurs à $90 \%$ à l'exception du tourteau de soja. Une faible digestibilité du soja a déjà été trouvée chez d'autres espèces de poissons (Hastings, 1969 ; Smith et al., 1980 ; Cho et al., 1982). La variabilité des résultats rapportés pour cette matière première est cependant assez importante. Les différents traitements subis par les tourteaux de soja disponibles dans le commerce n'éliminent pas complètement les facteurs antinutritionnels présents. L'importance des effets de ces facteurs reste à élucider afin de promouvoir l'emploi de cette matière première dans l'alimentation des poissons, surtout pour les espèces carnivores.

La disponibilité apparente de la somme des acides aminés essentiels ou totaux est du même ordre que le CUDA de l'azote total. Des résultats similaires ont été obtenus par Kaushik et Luquet (1976) et Hudon et De La Noüe (1985) chez la truite arc-en-ciel. Il est à noter que la disponibilité de certains acides aminés est systématiquement supérieure (lysine, arginine) ou inférieure (isoleucine) et cela indépendamment de la source. Néanmoins, des déviations par rapport à la digestibilité de l'azote total, propres à certains acides aminés, sont observées pour une matière première donnée. C'est le cas de l'acide aspartique de la farine de poisson, de l'isoleucine et la phénylalanine pour le tourteau de soja. II est donc nécessaire de connaître la disponibilité de chaque acide aminé en plus de la digestibilité de l'azote total, surtout si ces acides aminés risquent d'être limitants.

La digestibilité réelle des sources testées ne paraît pas différente de la digestibilité apparente. Cela est en accord avec les résultats trouvés par Nose (1967) et Rychly et Spannhof (1979) chez la truite et par Ogino et Chen (1973) chez la carpe. Ces auteurs ont montré que l'azote endogène n'influe pas sur la digestibilité des protéines quand ces dernières sont incorporées à des taux élevés dans le régime. Selon Ogino et Chen (1973), la différence entre digestibilité apparente et réelle devient importante quand l'ingéré azoté est inférieur à $100 \mathrm{mg}$ par $100 \mathrm{~g}$ de poisson et par jour. Lors de la présente expérience l'ingéré azoté était de l'ordre de $450 \mathrm{mg}$ par $100 \mathrm{~g}$ et par jour. L'absence de différence entre CUDA et CUDR a été également observée pour les acides aminés. Cela est en accord avec les résultats cités par Kaushik et Dabrowski (1983). La somme des acides aminés d'origine endogène est de $685 \mathrm{mg}$ par $100 \mathrm{~g}$ d'aliment. Des valeurs similaires ont été trouvées chez le poisson chat (Wilson et al., 1981) et la truite arc-en-ciel (Dabrowski et Dabrowska, 1981). Cette valeur est néanmoins supérieure à celle trouvée par Kaushik et Dabrowski (1983) chez la carpe. Le 
faible ingéré protéique expliquerait peut-être les différences entre la disponibilité apparente et réelle des acides aminés, observées par Wilson et al. (1981) chez le poisson chat.

Toutefois, l'absence de différence dans la présente étude peut également s'expliquer par les problèmes méthodologiques posés lorsqu'on veut quantifier la perte fécale endogène d'azote. En effet la quantité d'azote endogène excrétée est liée au régime utilisé et les résultats obtenus avec un aliment sans protéines ne sont pas forcément identiques à ceux d'un aliment normal, et cela d'autant plus que le régime protéiprive a été administré par gavage. A cela il faut rajouter les phénomènes de lessivage des fèces. Même si ce phénomène est minimisé par la méthode de collecte utilisée (Spyridakis et al., 1988), il n'est pas complètement éliminé et il est relativement plus important si la quantité d'azote dans les fèces est faible, comme c'est le cas pour le régime protéiprive.

\section{Conclusion.}

La présente étude montre que le calcul de la digestibilité de diverses matières premières par régression à partir d'un gradient de substitution permet une meilleure précision que l'extrapolation à partir d'un seul taux. Il s'agit néanmoins d'une méthode coûteuse et cela d'autant plus que le but ultime est l'établissement de tables de digestibilité pour toute une série de matières premières. Le recours à cette méthode paraît indispensable quand une grande précision est nécessaire et plus spécialement pour des sources protéiques susceptibles d'être incorporées à des taux élevés dans les aliments. Dans le cas contraire on peut se contenter d'un seul niveau d'inclusion à condition de le choisir assez élevé et de multiplier les mesures.

Les résultats obtenus montrent que la disponibilité des acides aminés est susceptible d'apporter des précisions complémentaires sur l'utilisation de certains d'entre eux; les difficultés de mesure ainsi que la faiblesse de la correction par l'azote endogène militent pour le choix des coefficients d'utilisation digestive apparente.

Reçu en avril 1988.

Accepté en juillet 1988.

Remerciements. - Le premier auteur exprime sa gratitude à la Fondation A.G. Leventis pour l'aide financière accordée à la réalisation de cette étude.

\section{Références}

ALLIOT E., 1982. Aspects nutritionne/s chez le loup Dicentrarchus labrax, en milieu contrôlé. Influence des régimes alimentaires et des facteurs de l'environnement sur le bilan azoté. Th. Doct. d'Etat. Univ. Aix Marseille II. Fac. Sci. de Luminy.

ALLIOT E., PASTOUREAUD A., PATROIS J., 1976. Etude de l'efficacité protéique de quelques farines de poissons pour l'alimentation du bar Dicentrarchus labrax. Tethys, 8, 335-338. 
ALLIOT E., PASTOUREAUD A., PELAEZ HUDLET J., METAILLER R., $1978 . \quad$ Utilisation des farines végétales et des levures cultivées sur alcanes pour l'alimentation du bar (Dicentrarchus labrax). Proc. World Symp. Finfish Nutr. Fishfeed Technol., Hamburg, Germany, 2, 229-238.

BOLIN D. W., KING R. P., KLOSTERMAN E. W., 1952. A simplified method for the determination of chromic oxid $\left(\mathrm{Cr}_{2} \mathrm{O}_{3}\right)$ when used as an index substance. Science, 116, 634-635.

CHO C. Y., SLINGER S. J. BAYLEY H. S. 1982. Bioenergetics of salmonid fishes, energy intake, expenditure and productivity. Comp. Biochem. Physiol., 73B, 25-41.

CHO C. Y., COWEY C. B., WATANABE T., 1985. Methodological approaches to research and development. In CHO C. Y., COWEY C. B., WATANABE T., Finfish nutrition in Asia. IDRC, Ottawa, Ont., $154 \mathrm{pp}$.

CHOUBERT G., DE LA NOÜE J., LUQUET P., 1982. Digestibility in fish : improved device for the automatic collection of faeces. Aquaculture, 29, 185-189.

DABROWSKI K., DABROWSKA H., 1981. Digestion of protein by rainbow trout (Salmo gairdneri R.) and absorption of amino acids within the alimentary tract. Comp. Biochem. Physiol., 69A, 99-111.

HASTINGS W. H., 1969. Nutritional score : 263-292. In Fish in research. NEUHAUS O. T., HALVER J. E., Acad. Press, New York-London.

HUDON B., DE LA NOÜE J., 1985. Amino acid digestibility in rainbow trout: influence of - temperature, meal size and type of food. World Maric. Soc., 16, 101-103.

KAUSHIK S., LUQUET P., 1976. Etude de la digestibilité des acides aminés de régimes à base de zéine chez la truite arc-en-ciel. Ann. Hydrobiol., 7, 11-19.

KAUSHIK S. J., DABROWSKI K., 1983. Nitrogen and energy utilisation in juvenile carp (Cyprinus carpio) fed casein, amino acids or a protein-free diet. Reprod. Nutr. Dévelop., 23, $741-754$.

KIM Y. K., 1974. Determination of true digestibility of dietary proteins in carp with chromic oxide containing diet. Bull. jap. Soc. sci. Fish. 40, 651-653.

MOORE S., SPACKMAN D. H., STEIN H. W., 1958. Chromatography of amino acids on sulfonated polystyrene resins. Analyt. Chem., 30, 1185-1190.

NOSE T., 1967. On the metabolic fecal nitrogen in young rainbow trout. Bull. Freshwater Fish. Res. Lab., 17, 97-105.

OGINO C., CHEN M. S., 1973. Protein nutrition in fish. III. Apparent and true digestibility of dietary proteins in carp. Bull. jap. Soc. Sci. Fish. 39, 649-651.

RYCHLY J., SPANNHOF L., 1979. Nitrogen balance in trout. I. Digestibility of diets containing varying levels of protein and carbohydrate. Aquaculture, 16, 39-46.

SMITH R. R., PETERSON M. C., ALLRED A. C., 1980. Effect of leaching on apparent digestion coefficients of feedstuffs for Salmonids. Prog. Fish Cult., 42, 195-199.

SPYRIDAKIS P., METAILLER R., GABAUDAN J., RIAZA A., 1988. Studies on nutrient digestibility in European sea bass (Dicentrarchus /abrax). 1. Methodological aspects concerning the faeces collection. Aquaculture (accepté pour publication).

WILSON R. P., ROBINSON E. H., POE W. E., 1981. Apparent and true availability of amino acids from common feed ingredients for channel catfish. J. Nutr., 111, 923-929.

YOSHIDA M., 1972. Evaluation of error of variance of metabolisable energy of feed ingredients. Jap. Poult. Sci., 9, 981 -985. 